\title{
mannenowe Painful numb hands
}

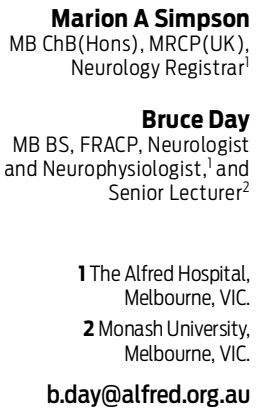

MJA 2011; 195: 388-391 doi: 10.5694/mjall.10900

\section{Previous article in this series "Blurred vision and pain in the eye" in MJA 2011; 195: 329-332}

The grading system for recommendations in this article is described in MJA 2011; 195: 328

Series Editors

Craig S Anderson MB BS, PhD, FRACP

Leo Davies MB BS, MD, FRACP

\begin{abstract}
Clare's story
Clare is a 54-year-old, right-handed pastry chef who presented with discomfort, numbness and tingling in both hands. Her symptoms had begun insidiously 9 months earlier, initially in the right hand, and had since become bilateral and severe, such that she was having difficulty performing her usual tasks at work. She described intermittent dull aching pain extending from the hands up to the elbows bilaterally, and pins and needles in all of the fingers. The symptoms occurred most commonly at work or while she was driving or holding a phone to talk. They were also waking her from sleep most nights. Shaking her hands provided some relief.

Clare's past medical history was otherwise unremarkable. Her weight was $85 \mathrm{~kg}$ (body mass index, $33 \mathrm{~kg} / \mathrm{m}^{2}$ ); she had gained $20 \mathrm{~kg}$ over the past 2 years after giving up ballroom dancing. She had recently been tested for diabetes as part of a routine check-up and was found to have normal glucose tolerance. She remembered her mother having similar symptoms in her hands, but did not recall any diagnosis being made.

On examination, Clare's hands appeared normal, with no evidence of muscle wasting and no weakness or incoordination. Sensory examination showed a subjective reduction of sensation in the index and middle fingers and thumb of the right hand. Reflexes were present and symmetrical. Prolonged wrist flexion (Phalen test; Box 1) reproduced symptoms of bilateral hand pain and paraesthesiae, but percussion of the median nerves at the wrist (Tinel test; Box 1) did not. The remainder of the neurological examination was normal.
\end{abstract}

\section{APPROACH TO THE PROBLEM}

The clinical history and examination in this case are highly suggestive of a peripheral nerve disorder. The intermittent paraesthesiae and discomfort in the digits supplied by the median nerve, together with nocturnal symptoms and relief provided by shaking, are classical for carpal tunnel syndrome. Carpal tunnel syndrome is commonly bilateral, although it may be asymmetrical and is usually worst in the dominant hand.

Many patients present with atypical symptoms. They may describe sensory symptoms and pain throughout the whole hand and forearm (as did Clare), finger swelling or loss of manual dexterity, or they may present with painless atrophy of the thenar eminence. They may simply complain of dropping objects.

Clare had several risk factors for carpal tunnel syndrome: an occupation involving repetitive use of the hands, female sex, positive family history, and obesity. Atrisk occupations are those that involve repetitive wrist

\section{Summary \\ - Carpal tunnel syndrome, resulting from median nerve compression at the wrist, is a common and often disabling mononeuropathy. \\ - Risk factors include female sex, family history, repetitive hand use, obesity, pregnancy and a variety of medical comorbidities including diabetes mellitus, rheumatoid arthritis, and other connective tissue diseases. \\ - In many cases, an accurate diagnosis can be reached on the basis of clinical history and supportive examination findings alone. \\ - Neurophysiological investigations are essential for confirming the diagnosis, assessing severity and excluding more generalised neuropathies, as well as providing a baseline preoperative index of median nerve function. \\ - Wrist splinting and local corticosteroid injection are effective treatments in the short term, but long-term data are lacking. \\ - Surgical (endoscopic or open) carpal tunnel release is effective and nearly always required to enable a return to work for patients with occupationally induced carpal tunnel syndrome.}

exertion, including factory work and trades such as carpentry or those involving use of vibrating tools.

\section{DIFFERENTIAL DIAGNOSES}

Differential diagnoses of hand numbness and paraesthesiae that should be considered in addition to carpal tunnel syndrome are summarised below.

Cervical radiculopathy is usually unilateral, accompanied by neck pain, and with symptoms provoked by neck movement, coughing, sneezing and the Valsalva manoeuvre. Normal results of a clinical neurological examination, with normal power and reflexes, would argue against this diagnosis.

Other neuropathies such as a more generalised neuropathic process, a peripheral neuropathy or mononeuritis multiplex would usually also include signs or symptoms in the legs.

Proximal median nerve compression can occur at the level of either the pronator teres, most often due to nerve entrapment between the two heads of the muscle, or the brachial plexus, due to an inflammatory process, trauma or mass lesions. It is important to identify these patients so that unnecessary surgery can be avoided. 


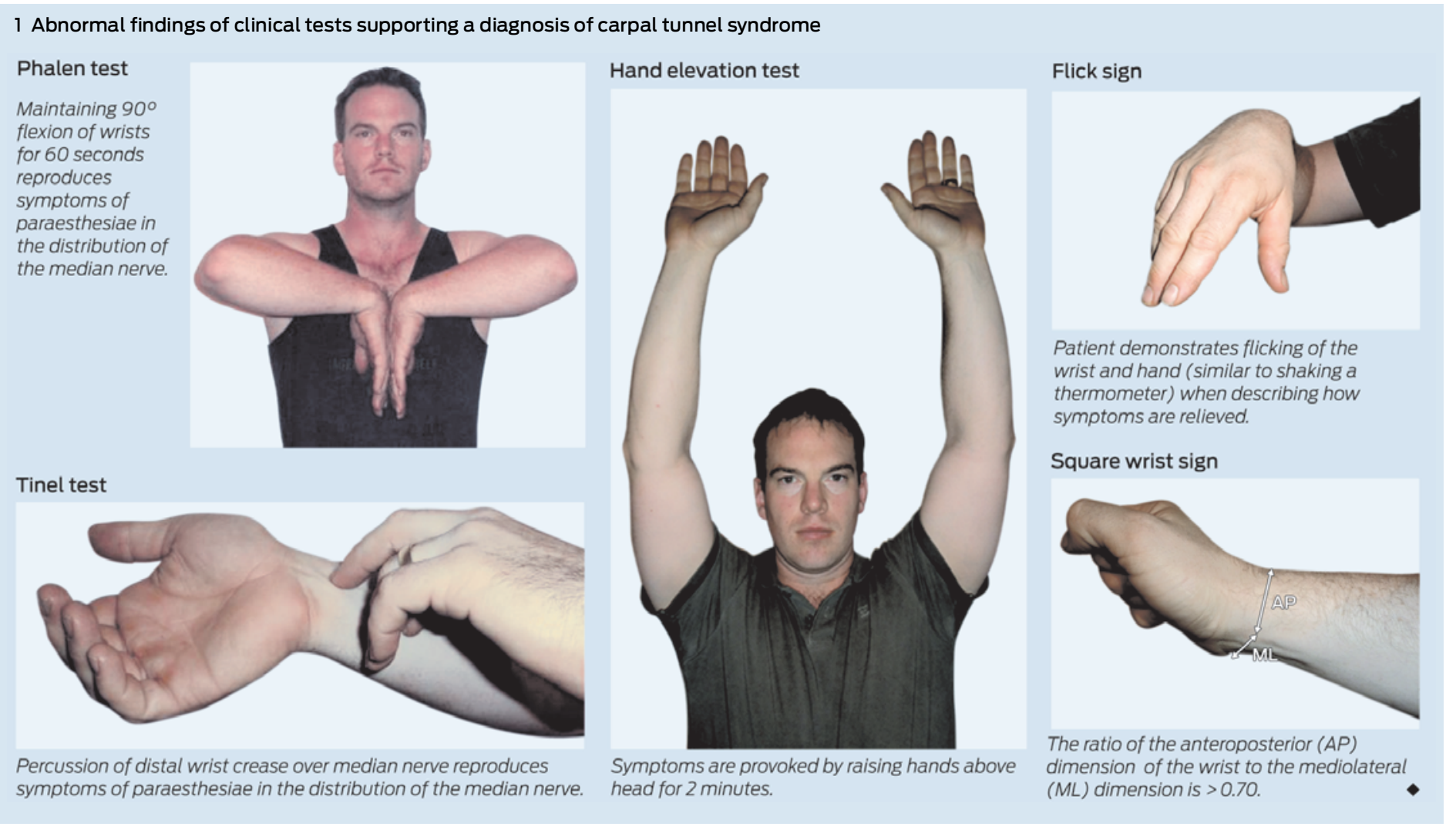

\section{APPROPRIATE USE OF INVESTIGATIONS}

Clinical tests: Several clinical tests, which vary in their diagnostic utility, ${ }^{1}$ can be used in the diagnosis of carpal tunnel syndrome (Box 1). There is wide variation in the reported sensitivity and specificity of these tests: the Phalen test, for example, one of the most commonly used, has a reported sensitivity of $10 \%-91 \%$ and specificity of $33 \%-86 \% .{ }^{1}$ In general, the Phalen and Tinel tests are the most widely used and are both more specific than sensitive.

Neurophysiological investigations: Although an accurate diagnosis of carpal tunnel syndrome can be made clinically in many cases, nerve conduction studies are essential for confirming the diagnosis and assessing severity. ${ }^{2}$ A baseline study before operative intervention can also assist with documenting a successful carpal tunnel release.

Electrodiagnostic tests are also useful for confirming or excluding an associated generalised neuropathy or mononeuritis multiplex, and for examining potential differential diagnoses such as cervical radiculopathy.

Results of Clare's nerve conduction studies are given in Box 2, with important positive findings highlighted. Her median motor nerve conduction study demonstrated a prolonged right median distal motor latency recording at the wrist.

Many neurophysiology laboratories grade severity of carpal tunnel syndrome according to a series of defined parameters, which can include the amplitude of the sen- sory action potential and the distal motor latency. However, criteria vary between laboratories, and there is often poor correlation of symptom severity with neurophysiological severity. Thus, severity grading may not be clinically useful and should be interpreted with caution.

Although useful, nerve conduction studies can produce false negative results in mild cases, or where intermittent ischaemia of the median nerve occurs without more prolonged demyelination. ${ }^{3}$ A repeat study after an interval of several months can help to identify such cases.

Radiological investigations: Ultrasonography and magnetic resonance imaging (MRI) can be used in the evaluation of carpal tunnel syndrome, to examine the dimensions of the median nerve at various points along its course and to detect macroscopic compression. However, commonly due to resource constraints with MRI and operator dependence with ultrasound, neither test is used in routine clinical practice at present. $^{3}$

Clare's likely diagnosis was bilateral (right worse than left) carpal tunnel syndrome, based on her typical symptoms and neurophysiological evidence of bilateral median neuropathy at the wrist. She had no evidence of an underlying systemic disorder such as rheumatoid arthritis or endocrinopathy. Her manual occupation and recent weight gain were predisposing or exacerbating factors (Box 3).

Given the persistent and disabling nature of her symptoms and the significant abnormalities demonstrated on her nerve conduction studies, Clare was counselled to pursue treatment. 


\section{How to interpret Clare's nerve conduction studies}

These tests confirm bilateral median neuropathies at the wrist, as evidenced by the following abnormalities (shown in blue):

A. Prolonged right median distal motor latency (DML) recording at the wrist, with normal conduction velocity in the forearm (recorded at elbow), suggesting focal abnormality at or distal to the wrist, rather than a diffuse process affecting the whole median nerve (see graph below). The left median DML at the wrist is just within normal limits.

B. Significant latency difference ( $\geqslant 1.0 \mathrm{~ms}$ ) on the left lumbrical median versus ulnar study, performed over the same distance for each nerve, suggesting abnormality affecting only the distal median nerve rather than a diffuse neuropathy.

C. Absent right median palmar potential - this has no localising value but does suggest median nerve abnormality.

D. Significant latency difference ( $\geqslant 0.4 \mathrm{~ms}$ ) on the left median versus ulnar palmar mixed nerve study, and bilateral median versus radial digit sensory studies, suggesting abnormality affecting only the median nerve.

E. Reduction in right median digit sensory action potential amplitude, suggesting more severe abnormality on this side, consistent with the patient's right hand dominance and initially right-sided symptoms.

Median motor nerve conduction study

\begin{tabular}{|c|c|c|c|}
\hline Nerve and site & Latency (ms) & Amplitude (mV) & $\begin{array}{c}\text { Velocity }(\mathrm{m} / \mathrm{s}) \\
\text { (RR, > 48) }\end{array}$ \\
\hline \multicolumn{4}{|c|}{ Right median - abductor pollicis brevis } \\
\hline Wrist (A) & $7.1(\mathrm{RR},<4.5)$ & $6.8(\mathrm{RR},>5)$ & \\
\hline Elbow & 9.1 & 6.6 & 51.4 \\
\hline \multicolumn{4}{|c|}{ Left median - abductor pollicis brevis } \\
\hline Wrist & 4.5 & 10.2 & \\
\hline Elbow & 8.1 & 9.8 & 59.3 \\
\hline \multicolumn{4}{|c|}{ Left carpal tunnel - lumbrical (B) } \\
\hline Median wrist & 5.2 & 2.0 & \\
\hline Ulnar wrist & 3.5 & 5.9 & \\
\hline \multicolumn{4}{|c|}{ Median sensory nerve conduction study (recording site: wrist) } \\
\hline Nerve and site & Latency (ms) & Amplitude $(\mu \mathrm{V})$ & $\begin{array}{c}\text { Velocity }(\mathrm{m} / \mathrm{s}) \\
(\mathrm{RR},>48)\end{array}$ \\
\hline \multicolumn{4}{|l|}{ Right median palmar } \\
\hline Median palm (C) & nr & nr & \\
\hline \multicolumn{4}{|l|}{ Left median palmar } \\
\hline Median palm (D) & 2.1 & 3.9 & 35.7 \\
\hline Ulnar palm & 1.7 & 2.0 & 54.9 \\
\hline \multicolumn{4}{|c|}{ Right carpal tunnel - digits } \\
\hline Ulnar digit $\mathrm{V}$ & 2.0 & 4.5 & 50.0 \\
\hline Median digit II (D, E) & 4.4 & $1.7(R R, \geqslant 6)$ & 29.5 \\
\hline Median digit I (D, E) & 3.6 & $2.5(R R, \geqslant 6)$ & 27.8 \\
\hline Radial digit I & 1.8 & 5.5 & 55.6 \\
\hline \multicolumn{4}{|c|}{ Left carpal tunnel - digits } \\
\hline Ulnar digit $\mathrm{V}$ & 1.8 & 4.7 & 61.1 \\
\hline Median digit II (D) & 3.1 & 12.3 & 39.3 \\
\hline Median digit I (D) & 3.0 & 6.8 & 30.5 \\
\hline Radial digit I & 1.5 & 6.0 & 60.0 \\
\hline
\end{tabular}

$\mathrm{RR}=$ reference range. $\mathrm{nr}=$ not recorded.

Nerve conduction studies for median motor nerve: normal and abnormal (Clare's) Normal
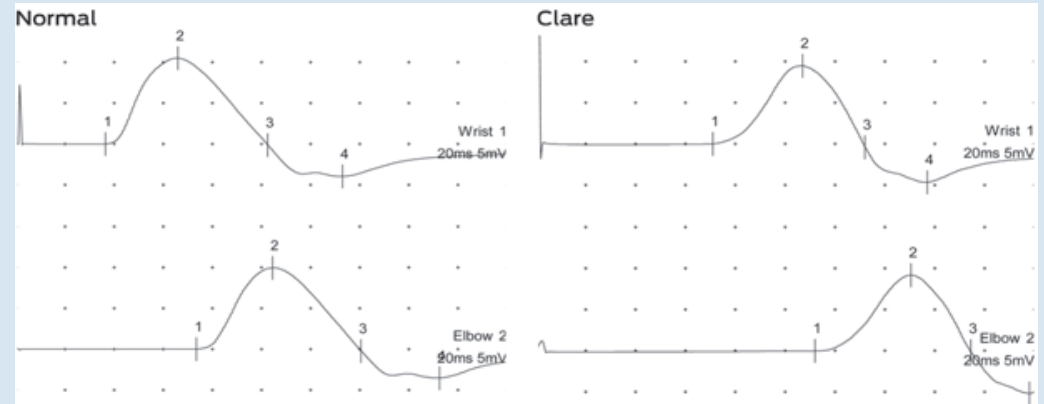

These studies were recorded at the abductor pollicis brevis, from stimulation at the wrist (top row) and elbow (bottom row). The number "7" indicates distal motor latency, which is $3.7 \mathrm{~ms}$ at the wrist in the normal study and $7.1 \mathrm{~ms}$ in Clare's study ( $R R,<4.5 \mathrm{~ms})$.

\section{Conservative management}

Several non-surgical treatments for carpal tunnel syndrome have been advocated, including rest, wrist splinting and medications (non-steroidal anti-inflammatory agents, diuretics, pyridoxine, and oral or intracarpal corticosteroids). Wrist splinting is the mainstay of conservative management. Splints worn at night, which keep the wrist in a neutral position and reduce nerve trauma due to flexion or extension during sleep, are often very effective for reducing waking caused by nocturnal symptoms in someone who has had a transient exacerbation after unusual activity (eg, performing home repairs) or during pregnancy.

Two Cochrane reviews have evaluated non-surgical treatments for carpal tunnel syndrome (Grade A evidence) ${ }^{5,6}$ One review (12 trials, 671 patients) found local corticosteroid injections in the region of the carpal tunnel to be more effective than placebo at 1 month (relative risk, $2.58 ; 95 \%$ CI $1.72-3.87$ ), and relative to oral steroids at 3 months, with no benefit shown for multiple injections over single injections. ${ }^{5}$ The other review (24 trials, 884 patients) found moderate evidence (from more than one randomised controlled trial) of short-term benefits (up to 3 months) from oral steroids, typically doses of $10-20 \mathrm{mg}$ of prednisolone per day, and limited evidence (from one randomised controlled trial) in favour of therapeutic ultrasound, splinting and carpal bone mobilisation, which involves physiotherapy-based techniques and stretching to "move" the bones and ligaments of the wrist. ${ }^{6}$ Few trials have examined long-term outcomes of non-surgical treatments.

\section{Surgical management}

Surgery for carpal tunnel syndrome may be performed by hand surgeons, orthopaedic surgeons, plastic surgeons or neurosurgeons. Two Cochrane reviews have evaluated surgical treatments of carpal tunnel syndrome (Grade A evidence). One review (four trials, 317 patients) demonstrated a clear benefit of surgery over splinting overall, although there was insufficient evidence regarding the effect of surgical treatment in the subgroup of milder cases or its relative efficacy over local steroid injection. ${ }^{7}$ The second review (33 trials, total number of patients not reported) compared endoscopic with open carpal tunnel release, and demonstrated equivalence in short-term and long-term outcomes between these techniques. ${ }^{8}$

Endoscopic decompression probably shortens the recovery period in uncomplicated carpal tunnel syndrome by about 6 days from an average of around 4 weeks, ${ }^{8}$ but it is not recommended in complicated cases where there is wrist deformity from arthritis or old fractures.

Prognosis after surgery can be difficult to predict, but a worse outcome is associated with longer duration of symptoms ( $>6$ months), older age, preoperative thenar wasting and poor response to local corticosteroid injection. ${ }^{9}$ Surgical failure has been associated with incomplete decompression of the carpal tunnel (when the incision has not been extended far enough into the palm). Horizontal incisions along the line of the wrist crease have been 


\section{Pathophysiology of carpal tunnel syndrome}

Carpal tunnel syndrome is the most common of the entrapment neuropathies, with a study in the United Kingdom finding an estimated annual incidence of 120 per 100000 in women and 60 per 100000 in men. ${ }^{4}$ It is also a disabling condition; occupationassociated carpal tunnel syndrome reportedly accounts for more work days lost than any other work-related injury or illness, with a median duration off work of 32 days. ${ }^{3}$

The carpal tunnel is formed by the carpal bones on the dorsal side and the flexor retinaculum on the palmar aspect. It contains the median nerve, together with the flexor tendons to the flexor digitorum profundus, flexor digitorum superficialis and flexor pollicis longus muscles. The size of the carpal tunnel varies with movement: wrist flexion causes the flexor retinaculum to move closer to the radius, and decreases its size. Extreme extension can also reduce the size of the carpal tunnel by moving the lunate bone towards the interior of the tunnel.

The most likely pathophysiology of carpal tunnel syndrome is compression of the median nerve in the narrow carpal tunnel, either by surrounding tissue or raised interstitial fluid pressure. However, the absolute size of the carpal tunnel has little bearing on the risk of developing symptoms.

Systemic conditions associated with carpal tunnel syndrome include diabetes mellitus, pregnancy, obesity, rheumatoid arthritis, other connective tissue diseases, hypothyroidism, amyloidosis and acromegaly.

Symptoms of carpal tunnel syndrome may be transient (eg, during pregnancy) or mild and non-disabling. The mechanism of

pregnancy-related carpal tunnel syndrome is believed to be related to increased fluid in tissues. It most often resolves after the infant's birth but can predict a higher risk of recurrent symptoms in later life. The mechanism by which obesity produces carpal tunnel syndrome is unknown. Weight loss has not been proven to relieve or resolve symptoms.

abandoned because of a high failure rate and postoperative dysaesthesiae from severing the small palmar cutaneous branch of the median nerve.

Occupationally induced carpal tunnel syndrome nearly always requires surgical decompression to enable a return to work. We usually advise those requiring surgery to anticipate being off work for 1 month. Usually, the symptoms are considerably if not completely better almost immediately after surgery, and patients can use their hands to perform their usual domestic tasks by the end of the first week. A return to heavy manual labour generally takes another 2-3 weeks. We also advise operating on one hand at a time, starting with the most symptomatic. Some surgeons recommend having surgery on both hands at the same time, but this approach requires the help of an understanding partner for toileting and other activities of daily living in the postoperative period.

\section{FACT OR FICTION?}

FACT: It is true that asymptomatic median neuropathy at the wrist, as an incidental finding during neurophysiological studies, is very common, particularly in patients with other neuropathies and arthritis of the hands. Such patients may also have wrist and joint pain.

FICTION: It is not true that all median neuropathy is carpal tunnel syndrome. Unless there is a clinical correlation showing that the neuropathy is due to median nerve compression (and carpal tunnel syndrome), patients are unlikely to respond to carpal tunnel release. Patients with severe median neuropathy, where the nerve is badly damaged and minimally functional, may no longer report symptoms and also may not benefit from surgery.

FINAL

\section{DIAGNOSIS}

Bilateral (right worse than left) carpal tunnel syndrome
Clare trialled wrist splinting for 1 month, but derived no benefit and was referred to a hand surgeon for carpal tunnel release. She underwent endoscopic release of her right carpal tunnel, with a good immediate result. She returned 3 months later to have the left hand treated, again with good relief of her symptoms. Clare successfully returned to work between the two procedures, performing light duties that involved minimal repetitive wrist movement, such as taking orders and answering phones rather than working on the production line, and returned to her full occupational duties 4 weeks after the second procedure. She did not require any further follow-up.

Competing interests: No relevant disclosures.

Provenance: Commissioned; externally peer reviewed.

1 D'Arcy CA, McGee S. The rational clinical examination. Does this patient have carpal tunnel syndrome? JAMA 2000; 283: 3110-3117.

2 Jablecki CK, Andary MT, Floeter MK, et al. Practice parameter: electrodiagnostic studies in carpal tunnel syndrome. Report of the American Association of Electrodiagnostic Medicine, American Academy of Neurology, and the American Academy of Physical Medicine and Rehabilitation. Neurology 2002; 58: 1589-1592.

3 Hui ACF, Wong S, Griffith J. Carpal tunnel syndrome [review]. Pract Neurol 2005; 5: 210-217.

4 Bland JD, Rudolfer SM. Clinical surveillance of carpal tunnel syndrome in two areas of the United Kingdom, 1991-2001. J Neurol Neurosurg Psychiatry 2003; 74: 1674-1679.

5 Marshall S, Tardif G, Ashworth N. Local corticosteroid injection for carpal tunnel syndrome. Cochrane Database Syst Rev 2002; (4): CD001554.

6 O'Connor D, Marshall S, Massy-Westropp N. Non-surgical treatment (other than steroid injection) for carpal tunnel syndrome. Cochrane Database Syst Rev 2003; (1): CD003219.

7 Verdugo RJ, Salinas RA, Castillo JL, Cea JG. Surgical versus non-surgical treatment of carpal tunnel syndrome. Cochrane Database Syst Rev 2008; (4): CD001552.

8 Scholten RJPM, Mink van der Molen A, Uitdehaag BMJ, et al. Surgical treatment options for carpal tunnel syndrome. Cochrane Database Syst Rev 2007; (4): CD003905.

9 Katz JN, Losina E, Amick BC. Predictors of outcomes of carpal tunnel release. Arthritis Rheum 2001; 44: 1184-1193. 